\title{
SY58-2 Symposium
}

\section{Genomics of Transporters: Implications to Drug Discovery, Development and Response}

\section{Kathy Giacomini}

Bioengineering and Therapeutic Sciences, UCSF, USA

Membrane transporters are critical determinants of drug action and disposition. In this presentation, I will describe genetic variation in membrane transporters in ethnically diverse populations, focusing in particular on polymorphisms in transporters that play a role in clinical drug response. The use of genomewide methods to discover endogenous substrates and biomarkers for transporters in the solute carrier superfamily (SLC) will be described. In addition, I will present recent studies in our laboratory focused on genetic variants in the organic anion transporter, OATP2B1, the organic cation transporter, OCT1 and the glucose transporter, GLUT2, which has been associated with response to the anti-diabetic drug, metformin, in large ethnically diverse populations. Overall, polymorphisms in transporters are increasingly being recognized for their role in drug disposition and therapeutic and adverse drug response. 